\title{
Cognitive behavioral therapy for somatic symptom disorders in later life: a prospective comparative explorative pilot study in two clinical populations
}

This article was published in the following Dove Press journal:

Neuropsychiatric Disease and Treatment

I September 2017

Number of times this article has been viewed

\author{
Michelle JH Verdurmen' \\ Arjan CVideler ${ }^{2}$ \\ Astrid M Kamperman ${ }^{3}$ \\ David Khasho ${ }^{4}$ \\ Christina M van der \\ Feltz-Cornelis ${ }^{4,5}$ \\ 'Department of Personality Disorders, \\ GGz Breburg, Tilburg, ${ }^{2}$ Department \\ of Geriatric Psychiatry, GGz Breburg, \\ Tilburg, ${ }^{3}$ Department of Psychiatry, \\ Erasmus University Medical Center, \\ Epidemiological and Social Psychiatric \\ Research Institute, Rotterdam, \\ ${ }^{4}$ Clinical Center of Excellence \\ for Body, Mind and Health, GGz \\ Breburg, Tilburg, ${ }^{5}$ Department of \\ Tranzo, Tilburg University, Tilburg, \\ The Netherlands
}

Background: Elderly patients with somatic symptom disorder (SSD) put a great burden on the health care delivery system. Cognitive behavioral therapy (CBT) is effective in adults with SSD. However, no studies have been conducted yet into CBT for SSD in later life.

Objectives: We explored the feasibility of CBT for SSD in the elderly.

Methods: This is a prospective pilot study comparing two outpatient specialty mental health settings for adults ( $<60$ years; $n=13$ ) and for elderly patients ( $\geq 60$ years; $n=9$ ) with SSD. Intervention was 18 structured, protocoled, and supervised CBT sessions. Outcomes were somatic symptoms, pain intensity, pain disability, quality of life, depressive symptoms, and generalized anxiety symptoms. Feasibility of the CBT intervention was explored with selfdeveloped questions, both for the therapists and the patients.

Results: Both therapists and elderly patients evaluated the treatment as positive. Somatic symptoms improved significantly in the adult group but not in the elderly group. There was a large, significant decrease in pain intensity and pain disability in elderly patients compared to the adults. Social functioning, vitality, and anxiety symptoms improved significantly in the adults. Presence of chronic medical conditions did not influence these results.

Conclusion: This study shows that CBT is feasible as a treatment for SSD in older adults and has encouraging results. Replication in an RCT is warranted.

Keywords: somatic symptom disorder, somatoform disorder, pain, elderly, medically unexplained symptoms

\section{Introduction}

Somatic symptom disorders (SSDs) place a great burden on the health care delivery system. An estimation of the prevalence of SSD in adults is $19 \% .{ }^{1} \mathrm{SSD}$ was introduced with $D S M-5^{1}$ to replace the $D S M-I V^{2}$ concept of somatoform disorders. The revised classification removes any reference to symptoms as medically unexplained ${ }^{3}$ and operationalizes the psychological concomitants of SSDs. ${ }^{4}$ The psychological concomitants of somatic symptoms are distressing and dysfunctional cognitions, emotions, and behaviors related to these bodily sensations, which are excessive and disproportional. Due to the physical presentation, individuals suffering with somatic symptoms initially seek help from a physician. Usually after many investigations, these individuals are referred to a psychiatrist. The prevalence of SSD in older adults is unknown, but considering the higher somatic comorbidity in later life, it is most likely that SSD is more common than the prevalence of somatoform disorders among the elderly, which is known to be somewhere in between 1.5 and $13 \% .{ }^{5,6} \mathrm{~A}$ recent study revealed that quality of life was similarly affected in both older patients with medically
Correspondence: Arjan C Videler Department of Geriatric Psychiatry, GGz Breburg, Piet Heinstraat I, Postbus 770, 5000 AT, Tilburg, the Netherlands Tel +3I 800162400 Email a.videler@ggzbreburg.nl 
explained and medically unexplained symptoms when corrected for somatization and hypochondriacal cognitions, which supports the relevance of the DSM-5 classification of SSD for older adults. ${ }^{7}$ The authors call for appropriate psychological treatment to improve quality of life in older SSD patients.

Cognitive behavioral therapy (CBT) has been found efficacious in the treatment of SSD in younger cohorts. ${ }^{8-10}$ We assume that SSD might even be more complex in older adults because of the higher somatic comorbidity in later life. CBT has been proven to have an almost similar efficacy in the treatment of depression and anxiety disorders in older adults up to the age of 80 years as in younger age groups. ${ }^{1-15}$ However, CBT as a treatment for SSD in older adults has not been explored yet.

\section{Objectives}

Because of the lack of research on treatment of SSD in older adults, the central objective of our study was to explore the feasibility of individual CBT for the treatment of SSD in older adults. We explored

1. Whether individual CBT in older adults is associated with a reduction in somatic symptoms compared to adults?

2. Whether individual CBT in older adults is associated with a reduction in pain intensity and disability, anxiety, and depressive symptoms and an increase in quality of life compared to adults?

3. How treatment effects of CBT in older adults compare with treatment effects of CBT in adults with SSD?

4. What the influence is of chronic somatic comorbidity?

We hypothesized CBT to be feasible in elderly SSD patients. We expected a reduction in somatic symptoms, pain, generalized anxiety, and depressive symptoms and an increase in quality of life in older adults.

\section{Methods}

\section{Participants}

All participants were patients with SSD who were referred between January 2015 and August 2016 to GGz Breburg, an institute for mental health care in the Netherlands. The elderly patients aged 60 years and older were treated in the Department of Geriatric Psychiatry. The adult patients younger than 60 years were treated in the Clinical Center of Excellence for Body, Mind and Health (CBMH). Participants were included if they met the criteria for an SSD diagnosis as assessed with a psychiatric DSM-5 interview. Exclusion criteria were major neurocognitive disorder (Mini-Mental State Examination ${ }^{16}$ score $\leq 23$ ), substance dependence, and IQ $<80$. Comorbidity with other mental disorders was allowed as long as SSD was the primary diagnosis. A concurrent somatic or psychological treatment was not allowed during the CBT treatment.

\section{Measures}

Feasibility of the intervention

Feasibility of the CBT intervention was explored with self-developed questions, both for the therapists and the patients. Questions concerned different qualitative aspects of the treatment, such as subjective efficacy of the treatment, susceptibility for the patient, time investment, and emotional burden.

\section{Physical Complaints Inventory (PCl)}

Somatic symptoms were assessed with the Dutch PCI, ${ }^{17}$ a 51-item self-report measure with a 4-point Likert scale (range 0-153). The reliability of the PCI is satisfactory (Cronbach's $\alpha$ : 0.88). ${ }^{18}$

\section{Brief Pain Inventory (BPI)}

Pain intensity and pain disability were assessed with two subscales of the BPI, ${ }^{19}$ the pain intensity subscale (four items), and limitations because of pain (seven items). ${ }^{20}$ The BPI has an 11-point Likert scale (range 0-10). Reliability of both subscales is satisfactory (pain intensity, Cronbach's $\alpha$ : 0.85; disability, Cronbach's $\alpha: 0.88) .{ }^{21}$

\section{Medical Outcomes Study 36-items Short Form Health Survey (SF-36)}

Quality of life was assessed with the Medical Outcomes Study SF-36, ${ }^{22}$ a 36-item self-report measure. This survey assesses the following eight dimensions: vitality, physical functioning, bodily pain, general health perceptions, physical role functioning, emotional role functioning, social role functioning, and mental health. Reliability and validity have been found to be satisfactory and good, respectively. ${ }^{23}$

\section{Patient Health Questionnaire-9}

Depressive symptoms were measured using the Patient Health Questionnaire-9 (PHQ-9), ${ }^{24}$ a 9-item self-report measure with a 4-point Likert scale. Reliability and validity are good. ${ }^{24}$

\section{Generalized Anxiety Disorder 7-item (GAD-7) scale} Generalized anxiety was assessed using the GAD-7 scale, ${ }^{25} \mathrm{a}$ 7-item self-report questionnaire with a 7-point Likert scale. Reliability and validity were found to be good. ${ }^{25}$ 


\section{Somatic comorbidity}

Presence of somatic comorbidity was determined by a physical examination prior to start of treatment. This included the physical record provided by the patients' GPs. Furthermore, the amount of somatic diseases was cross-checked with the Health Interview Questionnaire (HIQ) ${ }^{26}$ The HIQ is developed by the Dutch Central Agency of Statistics.

\section{Intervention}

The individual CBT was a CBT protocol for medically unexplained symptoms, which has been found efficacious in adults with an average age of 36 years. ${ }^{8}$ The main therapeutic techniques used included identification and modification of dysfunctional automatic thoughts and behavioral experiments aimed at breaking the vicious cycle of the symptoms and their consequences. The original treatment protocol varied between 6 and 16 weekly sessions, depending on the severity of the problem and the number of maintaining consequences. In this study, we decided for a treatment dosage of 18 weekly sessions, divided into sets of six sessions. After each set of sessions, the PCI and BPI were taken and discussed with the patients to monitor the treatment. Treatment could be terminated earlier, that is after each set of six sessions, if patient and therapist agreed that optimal symptom reduction was achieved, and this was supported by the outcome of the measures.

\section{Procedure}

Patients with a primary diagnosis of SSD, who met the inclusion criteria, were approached by the first author. Potential participants were fully informed about the study and gave written consent to participate. The elderly participants were examined by a geriatrician, and the adult patients were examined by physicians of the CBMH. Therapists were 11 psychologists, trained in CBT. Each therapist treated one to five patients (median: two patients per therapist). On average, the therapists had 8.5 years of experience in CBT. Experience did not differ between both treatment groups. Treatment integrity was monitored by means of supervision by the fourth author, who is experienced in the CBT protocol for SSD. Feasibility of the CBT intervention was explored 3 months after the treatment was finished.

\section{Design}

For assessing the feasibility, we explored the effects of the protocoled individual CBT treatment in a prospective comparative pilot study between a group of elderly SSD patients and a group of adults SSD patients. Measures were taken four times: at start of treatment and after 6, 12, and 18 CBT sessions. At baseline and end of treatment, all measures were taken. During the intermediate assessments, only the PCI and the BPI were taken. When it was decided to end treatment earlier because of optimal symptom reduction, the final assessment was done. The Medical Ethics Committee of Brabant and the local research ethics committee of GGz Breburg granted ethical approval.

\section{Statistics and data analysis}

To determine differences between the groups in sociodemographic and clinical characteristics, $\chi^{2}$ and Fisher's exact tests were conducted for categorical variables and $t$-tests and Mann-Whitney $U$-tests were conducted for (nonnormally distributed) continuous variables. In the latter case, median and interquartile range (IQR) were reported. Differences between baseline and end of treatment assessments were analyzed using Wilcoxon signed-rank tests for paired data. Furthermore, differences in change scores between the elderly and the adults were analyzed using Mann-Whitney $U$-tests. To interpret the size of the treatment effect, correlation coefficients $(r)$ were computed. An $r$ of 0.1 is considered as a small effect, 0.3 a medium effect, and $\geq 0.5$ a large effect. ${ }^{27}$

Finally, a mixed model analysis (including a random intercept) was conducted to examine the effect of CBT on number and intensity of somatic symptoms (time effect) and the differences in treatment effect of CBT between the elderly and the adults (time $\times$ group interaction). This analysis was repeated for pain intensity and pain disability. The results were adjusted for the number of chronic medical conditions and standardized somatic symptom score at baseline. We report $\beta$ s and the corresponding $95 \% \mathrm{CI}$ of the time effect and the interaction effect. Analyses were conducted on the full sample (intention to treat analysis). For this aim, missing data were imputed through last observation carried forward (LOCF) imputation. Next, analyses were repeated including only those participants who completed the treatment (completers analysis).

\section{Results \\ Patient flow}

Figure 1 shows the patient flow. After screening, 11 elderly and 16 adult patients were eligible. In the intention to treat analysis, 9 elderly patients and 13 adult patients were included. Finally, 7 elderly and 12 adults finished the study and were included in the completers analysis. The number of treatment sessions varied between 5 and 18 (median: 15 sessions). 

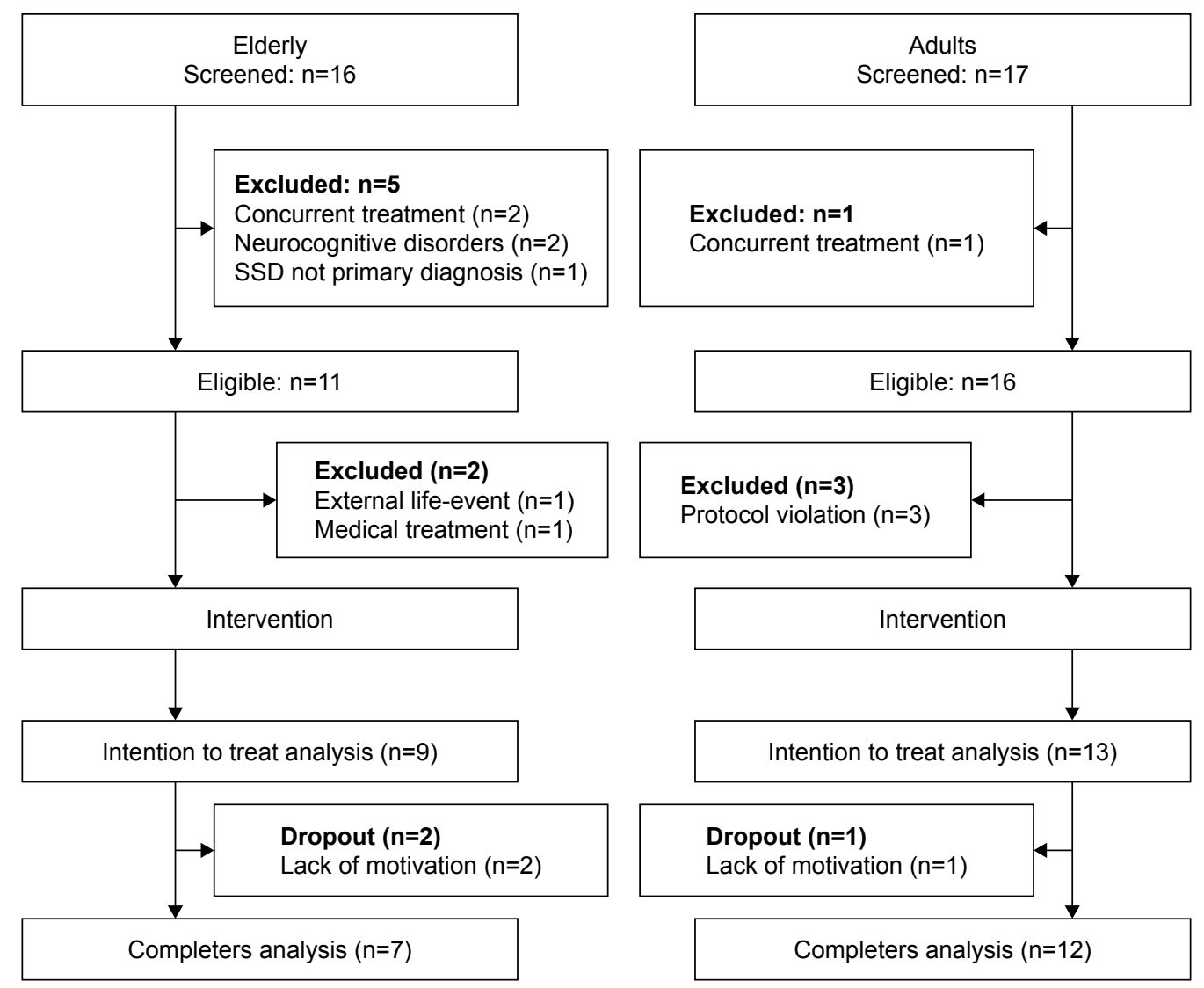

Figure I Patient flow.

Abbreviation: SSD, somatic symptom disorder.

The elderly patients received less sessions (median: 14; range: 6-18) than the adult patients (median: 16; range 6-18). This difference however is not significant (MWU[21] =72.0; $P=0.069$ ).

Table 1 describes the demographic and clinical characteristics of both groups at the start of treatment. All elderly patients reported at least one chronic medical condition, compared to only $62 \%$ of the adult patients $(8 / 13)$. None of the elderly patients had less than two comorbid medical conditions (range 2-4). These differences between the elderly group and the adult group were significant. Most elderly patients suffered from pain. Mood disorders were also prevalent. Most elderly patients used antidepressant, anxiolytic, and pain medications. A total of $30 \%$ of the elderly and $8 \%$ of the adults ended treatment earlier than the maximum of 18 sessions. This difference was not significant.

Table 2 shows the scores on all measures at start of treatment. Both groups reported high levels of somatic symptoms, pain, anxiety, and depressive symptoms and a low quality of life. There were no differences between the elderly and the adults except for lower general health perceptions.

\section{Treatment effects}

Tables 3 and 4 show the effects of the CBT treatment for the elderly and the adults. Small to medium effects were found for somatic symptoms in both groups. These treatment effects did not differ significantly between the groups. Reduction in somatic symptoms was significant for the adults.

Treatment effect on pain intensity was large for the elderly but small and not significant for the adults. The difference between these two effects was medium. Pain disability significantly decreased in the elderly group but not in the adult group.

Treatment effects on aspects of quality of life were small to medium. Again, on the pain intensity subscale, there was a significant medium effect in the elderly. The adults improved mainly on the subscales, such as social functioning and vitality. Differences on quality of life were not significant however between the two groups.

Both groups improved with a small-to-medium effect size on depressive symptoms and with a medium-to-large effect size on anxiety. Treatment effects on anxiety and depressive symptoms did not differ between the elderly and the adults. 
Table I Demographic and clinical characteristics of both groups

\begin{tabular}{|c|c|c|c|}
\hline & Elderly, n=9 & Adults, $n=13$ & $\begin{array}{l}\text { Statistical tests and } \\
P \text {-values }\end{array}$ \\
\hline Male, n (\%) & $5(56)$ & $2(15)$ & FET, $P=0.07$ \\
\hline Age, $M(S D)^{b}$ & $70.7(8.2)$ & $40.7(11.2)$ & $T(20)=6.843 ; P<0.00 I^{*}$ \\
\hline Education, $\mathrm{n}(\%)^{\mathrm{b}}$ & & & $\chi^{2}(3)=2.836 ; P=0.42$ \\
\hline Elementary school & $2(29)$ & $\mathrm{I}(8)$ & \\
\hline Lower vocational training & $3(43)$ & $4(31)$ & \\
\hline Higher secondary with vocational training & $I(14)$ & $6(46)$ & \\
\hline Higher education & I (I4) & $2(15)$ & \\
\hline Missing & 2 & & \\
\hline Primary complaint ${ }^{\mathrm{a}}$ & & & $\chi^{2}(2)=0.972 ; P=0.62$ \\
\hline Pain & $6(67)$ & $6(46)$ & \\
\hline Fatigue & $\mathrm{I}(\mathrm{II})$ & $3(23)$ & \\
\hline Others & $2(22)$ & $4(31)$ & \\
\hline \multicolumn{4}{|l|}{ Comorbid axis I disorders } \\
\hline Depression ${ }^{\mathrm{a}}$ & $4(44)$ & $4(31)$ & FET, $P=0.66$ \\
\hline Anxiety disorder ${ }^{\mathrm{a}}$ & $2(22)$ & $5(39)$ & FET, $P=0.65$ \\
\hline Others $^{\mathrm{a}}$ & $\mathrm{I}(\mathrm{II})$ & $4(31)$ & FET, $P=0.36$ \\
\hline No comorbiditya & $3(33)$ & $5(39)$ & FET, $P=0.99$ \\
\hline Comorbid personality disorder ${ }^{a}$ & & & $\chi^{2}(1)=1.67 I ; P=0.43$ \\
\hline No & $4(44)$ & $3(23)$ & \\
\hline Yes & $I(12)$ & $4(31)$ & \\
\hline Traits & $4(44)$ & $6(46)$ & \\
\hline Duration current SSD (years) ${ }^{\mathrm{a}}$ & & & FET, $P=0.99$ \\
\hline$<1$ & $7(78)$ & II (85) & \\
\hline$\geq 1$ & $2(22)$ & $2(15)$ & \\
\hline History of SSD & & & FET, $P=0.38$ \\
\hline First episode & $7(78)$ & $7(54)$ & \\
\hline Previous episodes & $2(22)$ & $6(46)$ & \\
\hline \multicolumn{4}{|l|}{ Somatic comorbidity } \\
\hline Yes $^{c}$ & $9(100)$ & $8(62)$ & $\chi^{2}(I)=4.480, P=0.03^{*}$ \\
\hline Number of diseases, median (IQR) $)^{d}$ & $3(2-3)$ & $\mathrm{I}(0-2)$ & $U=18.5, P=0.005^{*}$ \\
\hline $\begin{array}{l}\text { Number of appointments with health practitioners } \\
\text { over the last } 3 \text { months (Tic-P), median (IQR) }\end{array}$ & $13.5(\mid 1.5-18.5)$ & $13.0(4.5-22.5)$ & $U=27.5, P=0.42$ \\
\hline $\mathrm{N}_{\text {missing }}$ & I & 4 & \\
\hline Psychiatric admittance past 12 months $^{\mathrm{a}}$ & $\mathrm{I}(\mathrm{II})$ & I (8) & FET, $P=0.99$ \\
\hline \multicolumn{4}{|l|}{ Medication } \\
\hline Antidepressants ${ }^{\mathrm{a}}$ & $7(78)$ & $8(62)$ & FET, $P=0.65$ \\
\hline Anxiolytics $^{\mathrm{a}}$ & $5(56)$ & $5(23)$ & FET, $P=0.19$ \\
\hline Sleep medication ${ }^{\mathrm{a}}$ & $\mathrm{I}(\mathrm{II})$ & $2(15)$ & FET, $P=0.99$ \\
\hline Pain medication ${ }^{\mathrm{a}}$ & $4(44)$ & $2(15)$ & FET, $P=0.18$ \\
\hline Other medications $\mathrm{s}^{\mathrm{a}}$ & $\mathrm{I}(\mathrm{II})$ & $6(42)$ & FET, $P=0.16$ \\
\hline Dietary supplements ${ }^{\mathrm{a}}$ & $0(0)$ & $2(15)$ & FET, $P=0.94$ \\
\hline
\end{tabular}

Notes: Other comorbid axis I disorders: $A D(H) D$, minor neurocognitive disorder, and autism. Other medications: antipsychotics and anticonvulsants. aP-values obtained with Fisher's exact test; percentage between parentheses. ${ }^{b} P$-values obtained with independent $t$-test; percentage between parentheses. ${ }^{c} P$-values obtained with $\chi^{2}$ test; percentage between parentheses. ${ }^{d} P$-values obtained with Mann-Whitney U-test; percentage between parentheses. $*$ Significant to $P<0.05$.

Abbreviations: $A D(H) D$, Attention Deficit (Hyperactivity) Disorder; FET, Fisher's exact test; IQR, interquartile range; SSD, somatic symptom disorder; Tic-P, Toronto Institute for Contemporary Psychoanalysis.

The qualitative evaluation revealed that the therapists evaluated five out of six aspects of the CBT protocol as positive for the elderly patients. The elderly patients themselves evaluated all aspects of treatment as positive and rated the treatment with 8 out of 10 .

We applied a mixed model analysis to further analyze the effects of treatment ( $\mathrm{N}=23$ : ITT analysis), controlling for the presence of chronic medical conditions and the severity of complaints at the start of treatment. There appeared to be no independent effect of CBT on somatic symptoms ( $\beta=-0.75 ; 95 \%$ CI: -2.05 to $0.56, P=0.26$ ). Moreover, there was no significant difference between the course of treatment of the elderly and the adults $(\beta=-0.16 ; 95 \%$ CI: -2.45 to $2.13, P=0.89$ ).

Also for pain intensity, CBT did not have an independent effect ( $\beta=-0.34 ; 95 \% \mathrm{CI}:-2.15$ to $1.47, P=0.71$ ). For pain 
Table 2 Scores at start of treatment

\begin{tabular}{|c|c|c|c|}
\hline & Elderly $(n=9)$, median (IQR) & Adults $(n=13)$, median (IQR) & Difference between groups ${ }^{\mathrm{a}}$ \\
\hline Somatic symptoms $(\mathrm{PCl})$ & $25.0(5.0-37.5)$ & $15.0(9.5-18.5)$ & $U=43.5, P=0.324$ \\
\hline \multicolumn{4}{|l|}{ Pain (BPI) } \\
\hline Pain intensity & $21.0(21.0-24.0)$ & $23.0(17.0-29.0)$ & $U=70, P=0.47 \mathrm{I}$ \\
\hline Disability & $34.0(26.5-46.5)$ & $37.0(\mid 6.0-51.0)$ & $U=58, P=0.999$ \\
\hline \multicolumn{4}{|l|}{ Quality of life (SF-36) } \\
\hline General health perceptions & $14.0(\mid 1.0-18.5)$ & $19.0(16.5-22.0)$ & $U=91, P=0.030^{*}$ \\
\hline Emotional role functioning & $6.0(4.5-6.0)$ & $6.0(5.0-6.0)$ & $U=62, P=0.849$ \\
\hline Physical role functioning & $8.0(5.0-8.0)$ & $8.0(8.0-8.0)$ & $U=74, P=0.324$ \\
\hline Physical functioning & $21.0(13.0-25.0)$ & $17.0(\mid 2.5-26.0)$ & $U=54.5, P=0.794$ \\
\hline Mental health & $20.0(19.0-24.0)$ & $18.0(\mid 3.5-23.5)$ & $U=39.5, P=0.209$ \\
\hline Bodily pain & $7.00(5.5-8.5)$ & $8.00(5.0-10.5)$ & $U=75.5, P=0.262$ \\
\hline Social role functioning & $7.0(5.5-8.0)$ & $6.0(6.0-9.0)$ & $U=63, P=0.794$ \\
\hline Vitality & $15.0(\mid 1.5-18.0)$ & $20.0(\mid 7.0-21.5)$ & $U=88, P=0.05 \mathrm{I}$ \\
\hline Depressive symptoms (PHQ-9) & $13.0(6.0-19.0)$ & $11.0(9.5-18.5)$ & $U=70.5, P=0.43 \mathrm{I}$ \\
\hline Anxiety symptoms (GAD-7) & $14.0(8.0-19.5)$ & $11.0(6.5-13.0)$ & $U=39.5, P=0.209$ \\
\hline
\end{tabular}

Notes: ${ }^{a} P$-values obtained with Mann-Whitney $U$-tests. *Significant to $P<0.05$.

Abbreviations: BPI, Brief Pain Inventory; GAD-7, Generalized Anxiety Disorder 7-item scale; IQR, interquartile range; PCI, Physical Complaints Inventory; PHQ-9, Patient Health Questionnaire-9; SF-36, 36-items Short Form Health Survey.

intensity, there appeared to be a significant difference in the course of treatment between the elderly and the adults $(\beta=-3.54 ; 95 \%$ CI: -6.61 to $-0.47, P=0.024)$ in favor of the elderly. There was no independent treatment effect for pain disability ( $\beta=-1.73 ; 95 \% \mathrm{CI}:-4.17$ to $0.71 ; P=0.16$ ), and the difference in the course of treatment between both groups was not significant ( $\beta=-3.29 ; 95 \% \mathrm{CI}:-7.46$ to $0.89, P=0.12$ ). When we repeated these analyses for the patients who completed treatment, we found similar results. The course of treatment is represented in Figure 2A-C. The severity of symptoms is represented against the course of treatment. T1-T4 represent start of treatment, measurement after 6 weeks, after 12 weeks, and end of treatment, respectively.

\section{Discussion}

As this was the first study on treatment of SSD in later life, our main objective was to explore the feasibility of individual CBT in elderly patients with SSD compared to adults with SSD. Both patients and therapists evaluated the CBT protocol as positive for the elderly patients. The elderly patients rated

Table 3 Change scores (TI-T4) for elderly and adults

\begin{tabular}{|c|c|c|c|c|c|}
\hline & \multicolumn{2}{|l|}{ Elderly $(n=9)$} & \multicolumn{2}{|l|}{ Adults $(n=13)$} & \multirow{2}{*}{$\frac{\text { Elderly vs adults }}{\text { Test }^{\mathrm{b}}}$} \\
\hline & $\begin{array}{l}\text { Difference TI-T4, } \\
\text { median (IQR) }\end{array}$ & Test $^{\mathbf{a}}$ & $\begin{array}{l}\text { Difference TI-T4, } \\
\text { median (IQR) }\end{array}$ & Test $^{\mathbf{a}}$ & \\
\hline Somatic symptoms (PCl) & $-2.0(-6.0$ to 2.5$)$ & $Z=14.5, P=0.62$ & $-2.0(-6.5$ to 1.0$)$ & $Z=11.5, P=0.05$ & $U=50, P=0.60$ \\
\hline \multicolumn{6}{|l|}{ Pain (BPI) } \\
\hline Pain intensity & $-5.0(-15.0$ to -3.5$)$ & $Z=0, P=0.01 I^{*}$ & $0.0(-9.5$ to 6.0$)$ & $Z=32, P=0.58$ & $U=86, P=0.07$ \\
\hline Disability & $-9.0(-22.5$ to -3.0$)$ & $Z=0, P=0.012^{*}$ & $-4.0(-7.5$ to 3.0$)$ & $Z=24, P=0.24$ & $U=83.5, P=0.10$ \\
\hline \multicolumn{6}{|l|}{ Quality of life (SF-36) } \\
\hline General health perceptions & $0.0(-2.0$ to 2.5$)$ & $Z=10, P=0.92$ & $-1.0(-5.5$ to 1.0$)$ & $Z=14.5, P=0.18$ & $U=43.5, P=0.32$ \\
\hline Emotional role functioning & $0.0(-1.0$ to 0.0$)$ & $Z=0, P=0.10$ & $0.0(-1.0$ to 0.0$)$ & $Z=8.5, P=0.67$ & $U=66.5, P=0.60$ \\
\hline Physical role functioning & $0.0(-1.0$ to 0.0$)$ & $Z=4, P=0.7 I$ & $0.0(-2.0$ to 0.0$)$ & $Z=0, P=0.07$ & $U=5 \mid .5, P=0.65$ \\
\hline Physical functioning & $0.0(-5.0$ to 5.0$)$ & $Z=5, P=0.99$ & $-1.0(-2.5$ to 1.0$)$ & $Z=20, P=0.44$ & $U=45, P=0.39$ \\
\hline Mental health & $0.0(-4.5$ to 0.0$)$ & $Z=1.5, P=0.10$ & $-2.0(-3.5$ to 1.0$)$ & $Z=13, P=0.07$ & $U=59.5, P=0.95$ \\
\hline Bodily pain & $-1.0(-2.5$ to 0.0$)$ & $Z=0, P=0.04 I^{*}$ & $7.0(5.5$ to 9.0$)$ & $Z=9, P=0.06$ & $U=57.5, P=0.95$ \\
\hline Social role functioning & $0.0(-2.5$ to 0.0$)$ & $Z=0, P=0.07$ & $-2.0(-3.0$ to 0.0$)$ & $Z=8, P=0.045^{*}$ & $U=51.5, P=0.65$ \\
\hline Vitality & $0.0(-2.0$ to 0.0$)$ & $Z=2, P=0.27$ & $-1.0(-5.5$ to 0.0$)$ & $Z=0, P=0.007^{*}$ & $U=34, P=0.11$ \\
\hline Depressive symptoms (PHQ-9) & $0.0(-3.5$ to 2.0$)$ & $Z=9, P=0.75$ & $-4.0(-8.5$ to 1.5$)$ & $Z=15, P=0.06$ & $U=42.5, P=0.29$ \\
\hline Anxiety symptoms (GAD-7) & $-1.0(-9.5$ to 0.0$)$ & $Z=2, P=0.07$ & $-2.0(-5.5$ to 0.0$)$ & $Z=7, P=0.036^{*}$ & $U=58, P=1.00$ \\
\hline
\end{tabular}

Notes: ${ }^{\mathrm{T}}$ Test of paired TI-T4 measurement with Wilcoxon signed-rank tests. ${ }^{\circ}$ Test of change score between elderly and adults with Mann-Whitney U-tests. *Significant to $P<0.05$.

Abbreviations: BPI, Brief Pain Inventory; GAD-7, Generalized Anxiety Disorder 7-item scale; IQR, interquartile range; PCI, Physical Complaints Inventory; PHQ-9, Patient Health Questionnaire-9; SF-36, 36-items Short Form Health Survey. 
Table 4 Size of treatment effect (TI-T4) on each measure in correlation coefficient $(r)$

\begin{tabular}{|c|c|c|c|c|c|c|}
\hline & \multicolumn{2}{|l|}{ Elderly } & \multicolumn{2}{|l|}{ Adults } & \multicolumn{2}{|c|}{ Elderly vs adults } \\
\hline & $\begin{array}{l}\text { Results from } \\
\text { intention to } \\
\text { treat analysis }\end{array}$ & $\begin{array}{l}\text { Results from } \\
\text { completers } \\
\text { analysis }\end{array}$ & $\begin{array}{l}\text { Results from } \\
\text { intention to } \\
\text { treat analysis }\end{array}$ & $\begin{array}{l}\text { Results from } \\
\text { completers } \\
\text { analysis }\end{array}$ & $\begin{array}{l}\text { Results from } \\
\text { intention to } \\
\text { treat analysis }\end{array}$ & $\begin{array}{l}\text { Results from } \\
\text { completers } \\
\text { analysis }\end{array}$ \\
\hline Somatic symptoms (PCI) & 0.08 & 0.32 & 0.23 & 0.25 & 0.16 & 0.09 \\
\hline \multicolumn{7}{|l|}{ Pain (BPI) } \\
\hline Pain intensity & $0.55^{*}$ & $0.63^{*}$ & 0.11 & 0.12 & 0.38 & 0.44 \\
\hline Disability & $0.39 *$ & $0.58 *$ & 0.11 & 0.09 & 0.37 & $0.37^{*}$ \\
\hline \multicolumn{7}{|l|}{ Quality of life (SF-36) } \\
\hline General health perceptions & 0.01 & 0.08 & 0.25 & 0.25 & 0.25 & 0.27 \\
\hline Emotional role functioning & 0.18 & 0.34 & 0.04 & 0.03 & 0.18 & 0.26 \\
\hline Physical role functioning & 0.01 & 0.06 & 0.32 & 0.29 & 0.35 & 0.26 \\
\hline Physical functioning & 0.01 & 0.12 & 0.10 & 0.06 & 0.15 & 0.16 \\
\hline Mental health & 0.23 & 0.47 & 0.18 & 0.20 & 0.04 & 0.12 \\
\hline Bodily pain & $0.29 *$ & $0.42 *$ & 0.23 & 0.21 & 0.01 & 0.09 \\
\hline Social role functioning & 0.34 & 0.50 & $0.38 *$ & $0.38 *$ & 0.08 & 0.00 \\
\hline Vitality & 0.09 & 0.28 & $0.42 *$ & $0.44 *$ & 0.35 & 0.34 \\
\hline Depressive symptoms (PHQ-9) & 0.08 & 0.27 & 0.30 & 0.32 & 0.20 & 0.16 \\
\hline Anxiety symptoms (GAD-7) & 0.30 & $0.60 *$ & $0.37^{*}$ & $0.39 *$ & 0.03 & 0.09 \\
\hline
\end{tabular}

Note: *Significant to $P<0.05$.

Abbreviations: BPI, Brief Pain Inventory; GAD-7, Generalized Anxiety Disorder 7-item scale; PCI, Physical Complaints Inventory; PHQ-9, Patient Health Questionnaire-9; SF-36, 36-items Short Form Health Survey.
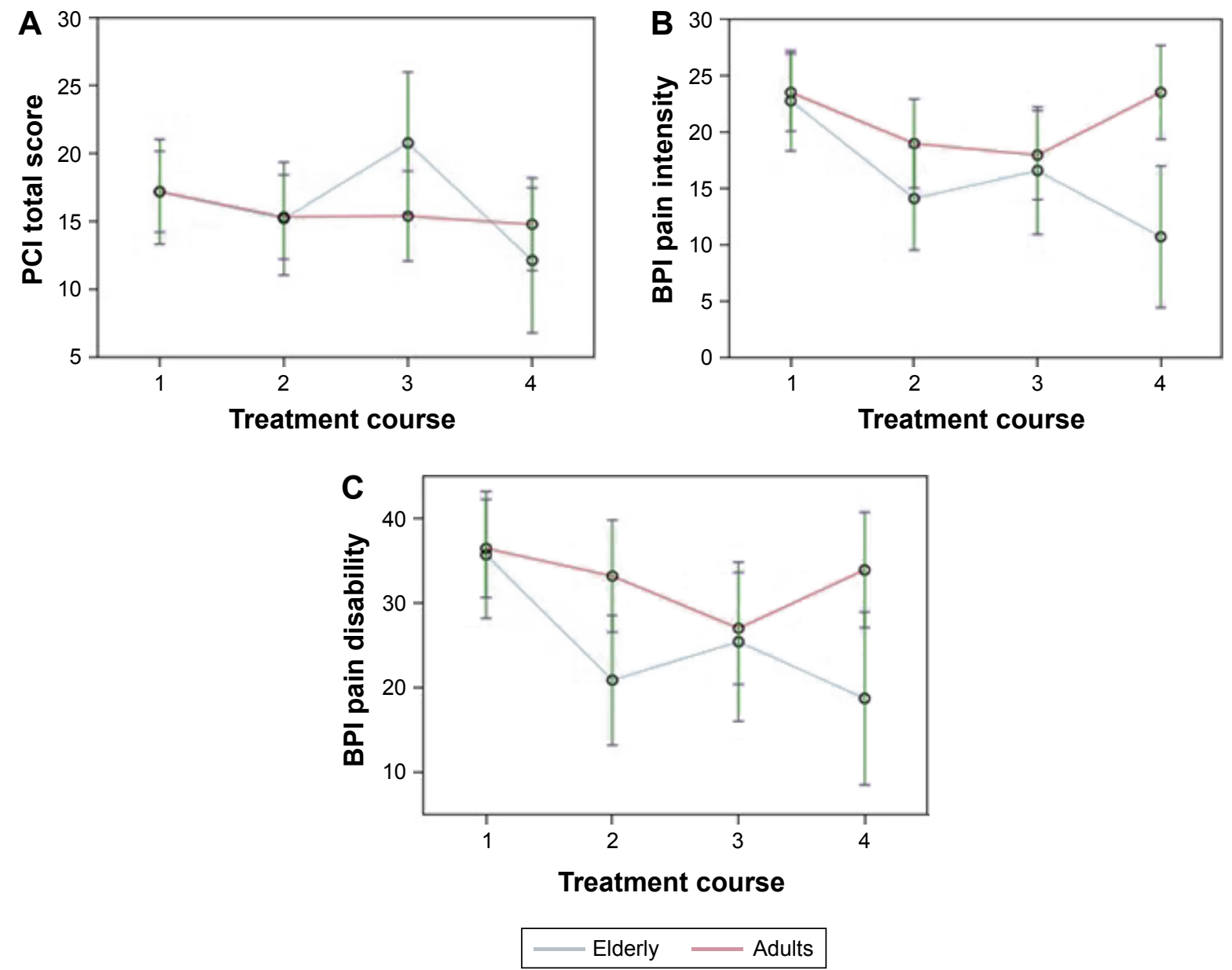

Figure 2 (A) Course of treatment for somatic symptoms. (B) Course of treatment for pain intensity. (C) Course of treatment for pain disability. Scores and $95 \%$ confidence interval estimated using mixed model-analysis (including random intercept), adjusted for number of chronic medical conditions and standardized somatic symptoms score at baseline. 
the treatment with 8 out of 10 . The observed effects on pain intensity, pain disability, and quality of life can be considered encouraging for the elderly. An explanation for the more limited effect on somatic symptoms might be that our elderly SSD patients suffered from more comorbid medical conditions than their younger counterparts, which can account for a smaller margin of improvement than among the younger SSD patients. A higher somatic comorbidity among elderly SSD patients was also found in other studies. ${ }^{5,6}$ However, we did not find that amount of chronic medical conditions influenced treatment outcome.

The higher effects of CBT on the pain measures in the elderly patients are a finding that warrants further research. Eisenberger ${ }^{28}$ describes that there is emerging evidence, which suggests that experiences of social pain (the painful feelings associated with social disconnection) are experienced in the same way as physical pain and that they rely on the same neurobiological substrates. This phenomenon could be more prevalent in later life, as older adults are less engaged socially. Such a finding is corroborated by the combined improvement of social engagement and pain in our study. We found that social functioning improved significantly in the elderly, which could support the hypothesis that when CBT improves social functioning and integration in elderly SSD patients, this might enhance treatment effect on pain.

Overall, the treatment effects in this study are similar to the small to medium effects, which were found in studies on CBT for medically unexplained symptoms in adults. ${ }^{10}$ The results of this pilot study are a first indication that CBT is feasible and associated with a positive treatment outcome treatment for SSD in later life. This is supported by the positive appraisal of the treatment by both patients and therapists.

\section{Limitations and strengths}

An important limitation of this study is its small sample size. Because of the sample size, the differences in outcomes between the two samples could possibly be accounted for by other reasons than the age of the participants. Central aim of this pilot study was exploring the feasibility of CBT for SSD in older adults. Our findings seem to justify replication of these results in a larger study. Another limitation concerns the observational design and lack of randomization, which can only show effects over time; we cannot rule out that the observed changes may have other explanations, like natural illness course or regression to the mean. We suggest conducting a randomized trial in future research. A third limitation is the lack of follow-up measurements to assess whether the effects hold over time. A fourth limitation is the sampling of patients from two different wards: there may have been biases in the referral patterns. Hence, the populations may not be fully comparable. A fifth limitation is that the "elderly" patients were defined by a relatively young age of 60 years. Although the mean age of the elderly eventually was $\sim 71$ years, we cannot be sure whether this treatment is as feasible for the old-old, aged $\geq 80$ years.

Strength of our study is the comparison of elderly patients with a group of adult patients with SSD to explore the differences in elderly SSD patients. Considering our findings, especially those on pain intensity and pain disability, we suggest to explore in future research whether pain is associated with social disconnection in later life and whether interventions that target this social connectedness enhance the treatment effect on pain in older adults.

\section{Conclusion}

This first study into the feasibility of CBT for the treatment of SSD in elderly patients provides us a preliminary indication that CBT is both applicable and potentially effective as a treatment for SSD in later life. Possibly, treatment of SSD in older adults should be focused more on the experiences and limitations of somatic symptoms than their reduction, considering a higher somatic comorbidity in later life. The role of social disconnection in pain and the options for enhancing treatment efficacy of SSD in later life deserve further research.

\section{Disclosure}

The authors report no conflicts of interest in this work.

\section{References}

1. American Psychiatric Association. Diagnostic and Statistical Manual of Mental Disorders. 5th ed. Arlington, VA: American Psychiatric Publishing; 2013.

2. American Psychiatric Association. Diagnostic and Statistical Manual of Mental Disorders. 4th ed. Washington, DC: American Psychiatric Association; 2000.

3. Dimsdale J, Creed F. The proposed diagnosis of somatic symptom disorders in DSM-V to replace somatoform disorders in DSM-IV - a preliminary report. J Psychosom Res. 2009;66(6):473-476.

4. Prior KN, Bond MJ. Somatic symptom disorders and illness behaviour: current perspectives. Int Rev Psychiatry. 2013;25(1):5-18.

5. Hilderink PH, Collard R, Rosmalen JGM, Oude Voshaar RC. Prevalence of somatoform disorders and medically unexplained symptoms in old age populations in comparison with younger age groups: a systematic review. Ageing Res Rev. 2013;12(1):151-156.

6. Wijeratne C, Brodaty H, Hickie J. The neglect of somatoform disorders by old age psychiatry: some explanations and suggestions for future research. Int J Geriatr Psychiatry. 2003;18(9):812-819.

7. Hanssen DJ, Lucassen PL, Hilderink PH, Naarding P, Oude Voshaar RC. Health-related quality of life in older persons with medically unexplained symptoms. Am J Geriatr Psychiatry. 2016;24(11):1117-1127. 
8. Speckens AE, Hemert AM, van Spinhoven P, Hawton KE, Bolk JH, Rooijmans HG. Cognitive behavioural therapy for medically unexplained physical symptoms: a randomised controlled trial. BMJ. 1995; 311(7016):1328-1332.

9. Kroenke K. Efficacy of treatment for somatoform disorders: a review of randomized controlled trials. Psychosom Med. 2007;69:881-888.

10. van Dessel N, den Boeft M, van der Wouden JC, et al. Nonpharmacological interventions for somatoform disorders and medically unexplained physical symptoms (MUPS) in adults. Cochrane Database Syst Rev. 2014;(11):CD011142.

11. Gould RL, Coulson MC, Howard RJ. Cognitive behavioral therapy for depression in older people: a meta-analysis and meta-regression of randomized controlled trials. J Am Geriatr Soc. 2012;60(10): 1817-1830.

12. Gould RL, Coulson MC, Howard RJ. Efficacy of cognitive behavioral therapy for anxiety disorders in older people: a meta-analysis and metaregression of randomized controlled trials. J Am Geriatr Soc. 2012; 60(2):218-229.

13. Hendriks GJ, Kampman M, Keijsers GP, Hoogduin CA, Oude Voshaar RC. Cognitive behavioral therapy for panic disorder with agoraphobia in older people: a comparison with younger patients. Depress Anxiety. 2014;31(8):669-677.

14. Gonçalves DC, Byrne GJ. Interventions for generalized anxiety disorder in older adults: systematic review and meta-analysis. $J$ Anxiety Disord. 2012;26(1):1-11.

15. Pinquart M, Duberstein PR, Lynness JM. Effects of psychotherapy and other behavioral interventions on clinically depressed older adults: a meta-analysis. Aging Ment Health. 2007;11(6):645-657.

16. Folstein MF, Folstein SE, McHugh PR. "Mini-mental state": a practical method for grading the cognitive state of patients for the clinician. J Psychiatr Res. 1975;12(3):189-198.

17. Hemert AM. Lichamelijke Klachten Vragenlijst [Physical Complaints Inventory]. Leiden: Leids Universitair Medisch Centrum; 2003.
18. De Waal MW, Arnold IA, Spinhoven P, Eekhof JAH, Assendelft WJJ, van Hemert AM. The role of comorbidity in the detection of psychiatric disorders with checklists for mental and physical symptoms in primary care. Soc Psychiatry Psychiatr Epidemiol. 2009;44(1):78-85.

19. Daut RL, Cleeland CS, Flanery RC. Development of the Wisconsin Brief Pain Questionnaire to assess pain in cancer and other diseases. Pain. 1983;17(2):197-210.

20. de Waal MW, Elzen WP, Achterberg WP, Gussekloo J, Blom JW. A postal screener for pain and need for treatment in older persons in primary care. J Am Geriatr Soc. 2014;62(10):1832-1837.

21. Tan G, Jensen MP, Thornby JI, Shanti BF. Validation of the Brief Pain Inventory for chronic non-malignant pain. Pain. 2004;5(2):133-137.

22. Ware JE, Sherbourne CD. The MOS 36-item short-form health survey (SF-36): I. Conceptual framework and item selection. Med Care. 1992;30(6):473-483.

23. Aaronson NK, Muller M, Cohen PD, et al. Translation, validation, and norming of the Dutch language version of the SF-36 health survey in community and chronic disease populations. J Clin Epidemiol. 1998; 51(11):1055-1068.

24. Kroenke K, Spitzer RL, Williams JB. The PHQ-9: validity of a brief depression severity measure. J Gen Intern Med. 2001;16(9):606-613.

25. Spitzer RL, Kroenke K, Williams JBW, Lowe B. A brief measure for assessing generalized anxiety disorder: the GAD-7. Arch Intern Med. 2006;166(10):1092-1097.

26. Central Agency of Statistics. Health Interview Questionnaire. The Hague: CBS; 1989.

27. Cohen J. Statistical Power Analysis for the Behavioral Sciences. 2nd ed. Hillsdale, NJ: Erlbaum; 1988.

28. Eisenberger NI. The pain of social disconnection: examining the shared neural underpinnings of physical and social pain. Nat Rev Neurosci. 2012; 13(6):421-434.
Neuropsychiatric Disease and Treatment

\section{Publish your work in this journal}

Neuropsychiatric Disease and Treatment is an international, peerreviewed journal of clinical therapeutics and pharmacology focusing on concise rapid reporting of clinical or pre-clinical studies on a range of neuropsychiatric and neurological disorders. This journal is indexed on PubMed Central, the 'PsycINFO' database and CAS,

\section{Dovepress}

and is the official journal of The International Neuropsychiatric Association (INA). The manuscript management system is completely online and includes a very quick and fair peer-review system, which is all easy to use. Visit http://www.dovepress.com/testimonials.php to read real quotes from published authors. 